\title{
Estabilidade de Fases em Blendas de Policarbonato- Poliestireno Avaliada por Micro-FTIR, Análise Térmica e Microscopia Eletrônica de Varredura
}

\author{
Rodrigo L. Oréfice, Wander L. Vasconcelos, Maria Antonieta S. Moraes \\ Laboratório de Engenharia de Polímeros e Compósitos, DEMM, UFMG
}

\begin{abstract}
Resumo: Nos últimos anos tem-se verificado uma acentuada desaceleração no desenvolvimento de novos homopolímeros, e nota-se um crescente interesse no estudo e desenvolvimento de processos que misturam os polímeros já existentes, dando origem às blendas poliméricas. Neste trabalho, blendas de poliestireno e policarbonato foram preparadas por extrusão em diferentes proporções. O processo de separação de fases foi estudado através da combinação de ferramentas analíticas como microscopias de infravermelho (micro-FTIR), óptica, eletrônica de varredura, e análise térmica. Resultados obtidos a partir das microscopias eletrônica e óptica permitiram definir as correlações entre morfologia e grau de estabilidade das fases do sistema. A composição e proporção relativas das fases das blendas foram determinadas usando microscopia de infravermelho. Tais resultados foram utilizados no cálculo do parâmetro de interação polímero-polímero de Flory-Huggins.
\end{abstract}

Palavras-Chave: Blendas poliméricas, policarbonato, poliestireno, microscopia de infravermelho.

\section{Phase Stability of Polycarbonate-polystyrene Blends Evaluated by Micro-FTIR, Thermal Analyses and Scanning Electron Microscopy}

\begin{abstract}
In the last decade, a progressive reduction on the development of new homopolymers could be observed, while processes, such as blending, that combine commercially available polymers were emphasized. In this work, polycarbonatepolystyrene blends were extruded in compositions from $0 \%$ to $100 \%$ of polycarbonate in weight. The phase separation process was studied by combining a series of analytical techniques such as scanning electron, optical and infrared microscopy (micro-FTIR). Electron and optical microscopy allowed the determination of a correlation between phase morphology and the degree of phase stability of the system. The composition of the phases and information on phase stability were determined by using micro-FTIR. The Flory-Huggins interaction parameter could also be calculated from the microFTIR data.
\end{abstract}

Keywords: Polymer blends, polycarbonate, polystyrene, infrared microscopy.

\section{Introdução}

Há um crescente interesse na pesquisa e desenvolvimento de processos que misturam homopolímeros já existentes, uma vez que isto permite novas combinações de propriedades e novas aplicações usando produtos já pesquisados e consagrados ${ }^{[1]}$.

Blendas poliméricas são misturas físicas de dois ou mais polímeros, sem que haja ligação química entre eles. Elas são bastante interessantes devido ao efeito cooperativo decorrente da mistura que se revela como melhoria das propriedades, em relação às propriedades dos homopolímeros ${ }^{[2]}$. Quando se trata de mistura de polímeros, a massa pode se apresentar como um sistema homogêneo, unifásico, ou como um sistema heterogêneo, multifásico, e as propriedades dependem da composição do sistema, da compatibilidade de seus ingredientes, do processamento e da morfologia apresentada pelas fases ${ }^{[3]}$. Um dos requisitos para o sucesso de uma blenda na aplicação final é a qualidade e homogeneidade da mistura polimérica ${ }^{[4]}$. Várias tentativas têm sido feitas para conseguir uma blenda com maior grau de homogeneidade, já que a quase nula contribuição entrópica de mistura acentua a tendência à imiscibilidade ${ }^{[4]}$.

Blendas com policarbonato e diferentes polímeros tem sido alvo de diversas pesquisas, na expectativa de mudanças na transparência óptica, estabilidade térmica e propriedades mecânicas dos polímeros dispersos ${ }^{[5-7]}$, uma vez que o policarbonato tem excelentes propriedades mecânicas, sendo a resistência ao impacto maior que qualquer outro termoplástico de engenharia e sua transparência comparável à do vidro de silicato, propriedades estas bastante desejáveis em várias aplicações comerciais.

O objetivo deste estudo foi investigar o processo de separação de fase da blenda policarbonato-poliestireno em blendas com três composições diferentes, preparadas por extrusão. Microscopia de infravermelho (micro-FTIR), 
microscopia óptica e microscopia eletrônica de varredura foram usadas como ferramentas na identificação, avaliação da composição e proporção relativa das fases. A determinação da composição das fases por micro-FTIR permitiu ainda avaliar o parâmetro de interação polímero-polímero de Flory$\operatorname{Huggins}\left(\chi_{1,2}\right)$ para as diferentes blendas.

\section{Experimental}

Para o processamento da blenda foi utilizada uma extrusora (SEIBT-Modelo Es/25), que teve o seguinte perfil de temperatura: $200^{\circ} \mathrm{C}$ (zona de alimentação), $220^{\circ} \mathrm{C}$ (zona 2), $240{ }^{\circ} \mathrm{C}$ (zona 3) e $240^{\circ} \mathrm{C}$ (matriz). Os polímeros (policarbonato - GE - massa molar $=25000 \mathrm{~g} / \mathrm{mol}$, poliestireno BASF, massa molar $=20000 \mathrm{~g} / \mathrm{mol}$ ) foram pesados, misturados e extrudados a fim de conseguir blendas com diferentes composições: $0,25 \%$, $50 \%, 75 \%$ e $100 \%$ em massa de policarbonato.

A caracterização das amostras produzidas via extrusão foi feita através das seguintes técnicas: FTIR, espectroscopia na região do infravermelho - Perkim-Elmer Paragon 1000; DSC, calorimetria exploratória diferencial - Shimadzu DSC 50; MEV, microscopia eletrônica de varredura - (JEOL) e microscopia de infravermelho.

FTIR: Filmes finos de cada blenda foram produzidos a partir da dissolução das blendas em clorofórmio e vazamento em placas de vidro. Os filmes foram, então, descolados das placas de vidro para serem investigados. Os filmes foram colocados diante do feixe de infravermelho (Perlin-Elmer, Paragon 1000) e espectros foram coletados na faixa entre $4000 \mathrm{~cm}^{-1} \mathrm{e} 400 \mathrm{~cm}^{-1}$ (número de varreduras por amostra $=4$ ). Acoplado ao espectrômetro, foi usado um microscópio óptico dotado de feixe de infravermelho que permitiu analisar puntualmente as amostras, definindo locais de heterogeneidades microestruturais, as quais, em seguida, foram analisadas por FTIR.

DSC: Foram obtidas por corte, pequenos exemplares das três blendas e dos dois homopolímeros pesando em torno de $10 \mathrm{mg}$ a $12 \mathrm{mg}$ cada. Cada exemplar foi inserido em cadinhos de alumínio. $\mathrm{O}$ equipamento foi programado da seguinte maneira: taxa de aquecimento $=20^{\circ} \mathrm{C} / \mathrm{min}$; temperatura final $=200^{\circ} \mathrm{C}$; atmosfera de nitrogênio; amostra referência $=$ óxido de alumínio.

MEV: As amostras foram fraturadas em nitrogênio líquido, para observação da superfície de fratura. As superfícies de fratura foram recobertas com fina camada de $\mathrm{Au}-\mathrm{Pd}$, para permitir a observação no MEV, uma vez que as amostras não são condutoras. A microestrutura de cada amostra foi analisada com o uso de três aumentos: 50X, 100X, 1000X.

\section{Resultados e Discussão}

A Figura 1 mostra o fluxo de produção da extrusora em função da composição da blenda em duas rotações (10 rpm e $50 \mathrm{rpm}$ ). Os resultados mostraram que as variações em viscosidade e massa molar, eventualmente presentes, não produzem diferenças significativas no fluxo da extrusora. $\mathrm{O}$

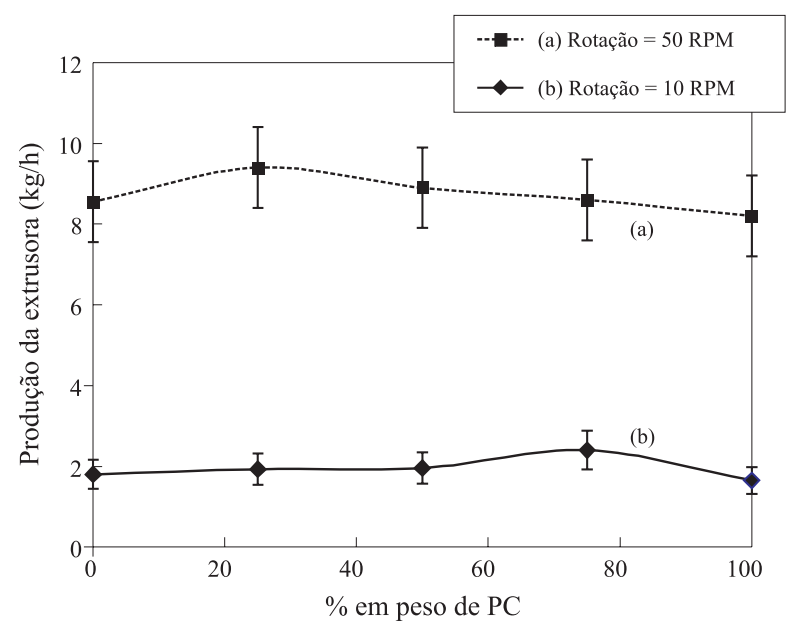

Figura 1. Fluxo de produção da extrusora usada na formulação da blenda PS-PC.

aumento da velocidade de rotação em cinco vezes (10 rpm e $50 \mathrm{rpm}$ ) provocou mudanças proporcionais no fluxo. As barras de erro indicam a dispersão das medidas de produção para um intervalo de confiança de $5 \%$.

A Figura 2 exibe a região de absorção no infravermelho entre $2700 \mathrm{~cm}^{-1} \mathrm{e} 3300 \mathrm{~cm}^{-1}$ para as diferentes composições da blenda. Tal região diz respeito às bandas de absorção dos grupamentos metileno e metila localizados entre $2800-3000 \mathrm{~cm}^{-1}$ e em grupos aromáticos (3000-3100 $\left.\mathrm{cm}^{-1}\right)$. A banda de absorção em $2927 \mathrm{~cm}^{-1}$ é característica das ligações $-\mathrm{CH}_{2}$ - do poliestireno, enquanto a banda de absorção a $2969 \mathrm{~cm}^{-1}$ é típica dos grupos $-\mathrm{CH}_{3}$ - do policarbonato. A razão entre as bandas de absorção a $2927 \mathrm{~cm}^{-1}$ e $2969 \mathrm{~cm}^{-1}$ pode ser relacionada linearmente com

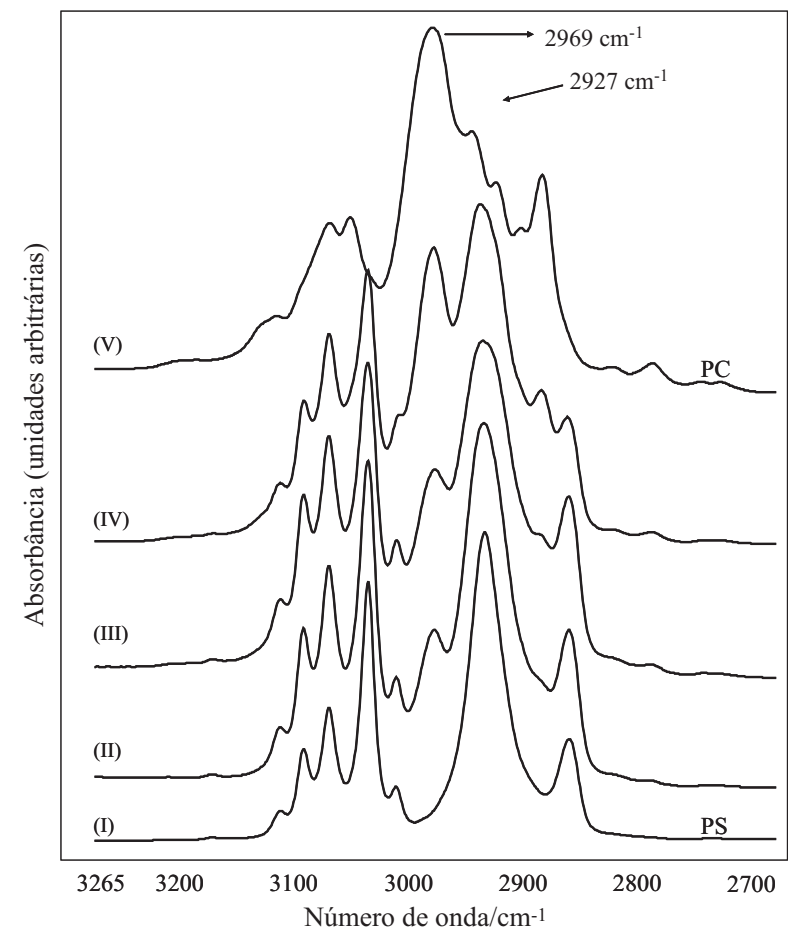

Figura 2. Espectros na região do infravermelho das várias composições da blenda PS-PC. (I) $100 \%$ PS; (II) $75 \%$ PS; (III) $50 \%$ PS; (IV) 25\% PS; (V) $0 \%$ PS. 


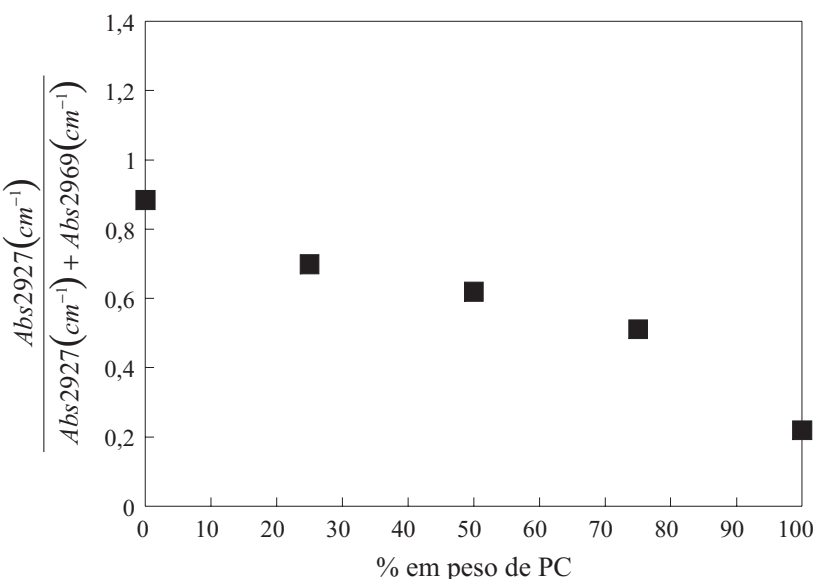

Figura 3. Razão entre as bandas de absorção de 2927 e $2969 \mathrm{~cm}^{-1}$ em função da composição da blenda PC-PS.

a composição da blenda, o que sugere um método eficiente para a deteç̧ão rápida da proporção entre os componentes da blenda a partir das informações de FTIR (Figura 3).

A Figura 4 revela as curvas DSC para as diversas composições da blenda PC-PS. As temperaturas de transição vítrea estão claramente demarcadas nas curvas e correspondem as temperaturas de transição vítrea $\left(\mathrm{T}_{\mathrm{g}}{ }^{\prime} \mathrm{s}\right)$ dos homopolímeros $\left(\mathrm{PS}=105^{\circ} \mathrm{C}\right.$ e $\left.\mathrm{PC}=155^{\circ} \mathrm{C}\right)$, o que indica um processo de separação de fase.

A morfologia das blendas produzidas pode ser estudada através da Figura 5, a qual mostra fotomicrografias obtidas por microscopia eletrônica de varredura da superfície de fratura de blendas. A Figura 5-a revela a superfície de fratura da blenda com composição $50 \%$ em peso de PS e mostra uma tendência de conectividade entre as fases. Enquanto isso, a Figura 5-b mostra a superfície de fratura da blenda contendo $25 \%$ em peso de PC e evidencia a presença de uma fase dispersa (esferas de PC) numa matriz de PS.

As várias blendas processadas foram investigadas via micro-FTIR visando a determinação da composição das fases presentes. As Figuras 6 e 7 exemplificam a metodologia e os resultados obtidos. Na Figura 6, a micrografia da blenda com $75 \%$ de PS é exibida e permite a observação do processo de separação de fase. Cada uma das fases foi então analisada

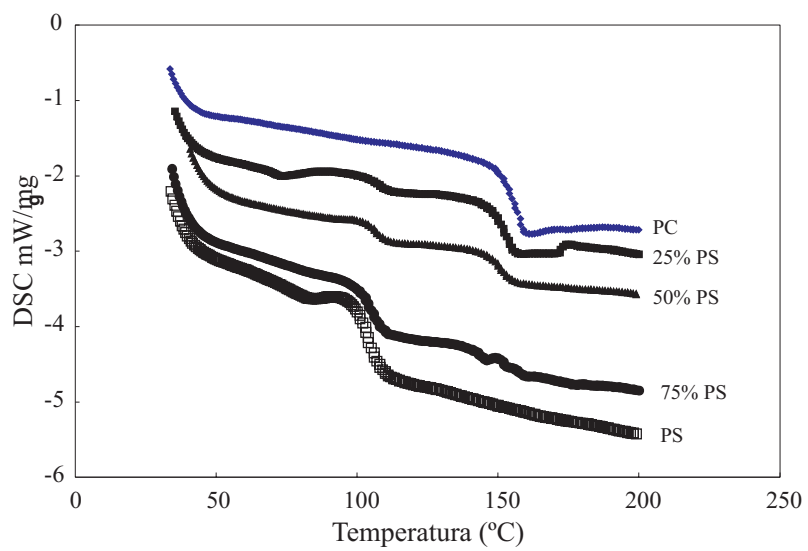

Figura 4. Curvas DSC para as várias blendas PC-PS: (I) 100\% PS; (II) $75 \%$ PS; (III) $50 \%$ PS; (IV) $25 \%$ PS; (V) $0 \%$ PS (PC).

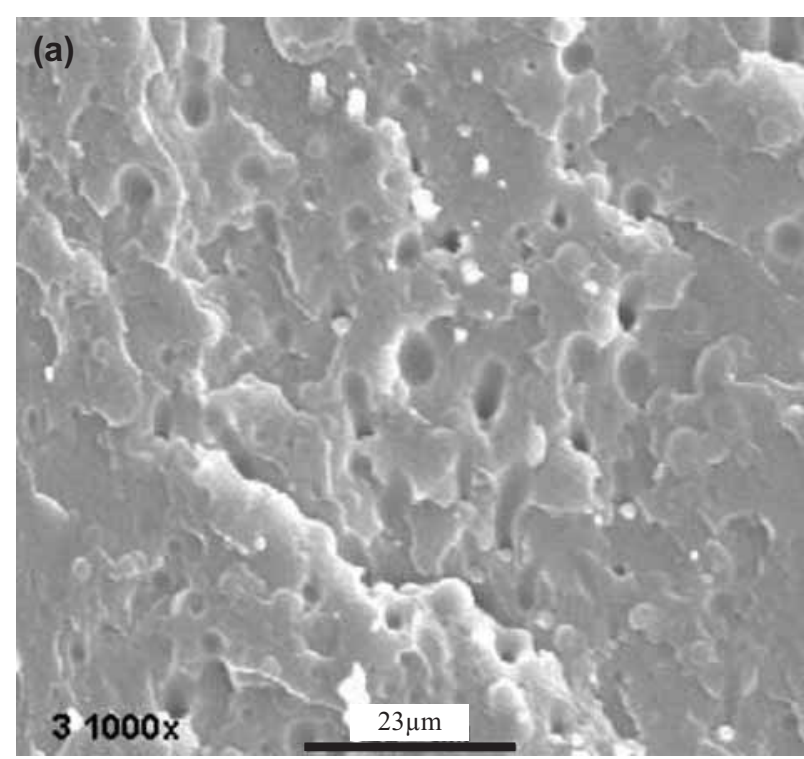

(a)

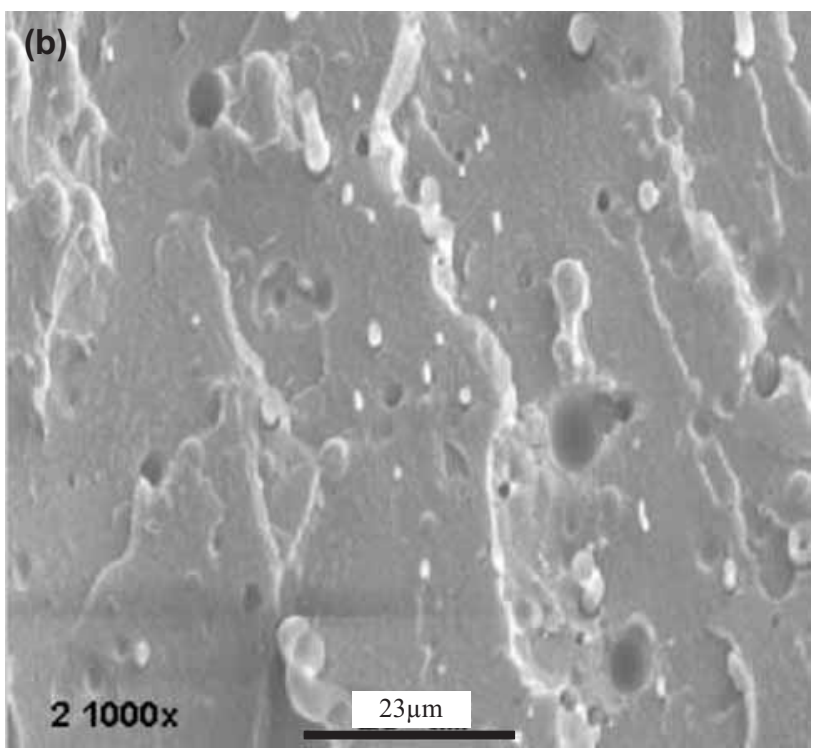

(b)

Figura 5. Fotomicrografias obtidas via MEV das blendas: (a) 50\% PS e (b) $25 \% \mathrm{PC}$

puntualmente através da micro-FTIR. A Figura 7 exibe os espectros na região de absorção no infravermelho para os homopolímeros (Figuras 7-a e 7-b) e para a blenda 75\% PS analisada de forma global (Figura 7-c). Nesta figura, ainda são mostrados os espectros coletados na fase dispersa (Figura 7-d) e na matriz (Figura 7-e) da blenda com 75\% PS. Como dito anteriormente, a banda de absorção em $2927 \mathrm{~cm}^{-1}$ é característica das ligações alifáticas $-\mathrm{CH}_{2}$ - do poliestireno, enquanto a banda de absorção a $2969 \mathrm{~cm}^{-1}$ é típica dos grupos $-\mathrm{CH}_{3}$ - do policarbonato. A razão entre as bandas de absorção a 2927 e $2969 \mathrm{~cm}^{-1}$ pode ser relacionada com a composição das fases.

Os resultados obtidos a partir da microscopia de infravermelho puderam ser convertidos em valores de composição das fases a partir de curvas de correlação, como a 


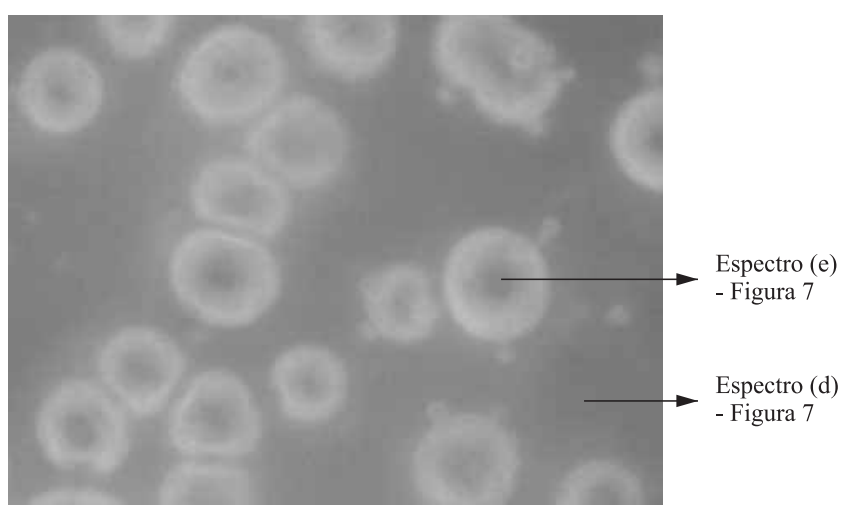

Figura 6. Micrografia da blenda contendo $75 \%$ em peso de PS (aumento 100X).

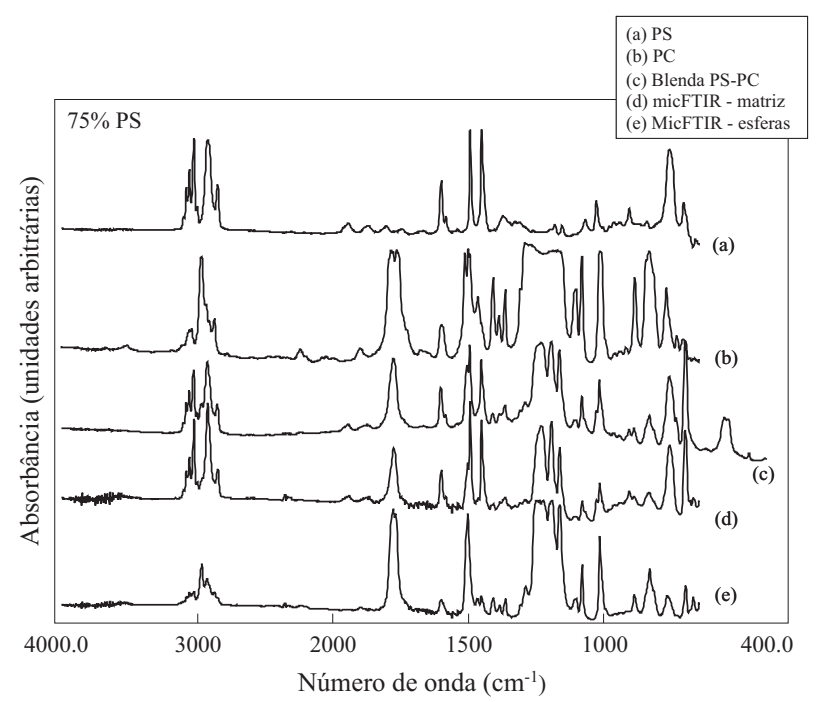

Figura 7. Espectros na região do infravermelho para a blenda contendo $75 \%$ de PS

exibida na Figura 3. As frações em peso foram convertidas para fração volumétrica usando os valores de densidade dos homopolímeros (densidade PS $=1,05 \mathrm{~g} / \mathrm{cm}^{3}$ e densidade do $\left.\mathrm{PC}=1,15 \mathrm{~g} / \mathrm{cm}^{3}\right)$. Os valores de composição foram então usados no cálculo das proporções relativas entre as fases (através de um balanço de massa) e na obtenção dos parâmetros de interação de Flory-Huggins para o sistema. Os resultados gerais exibidos na Tabela 1 mostraram uma tendência de enriquecimento em poliestireno das fases ricas em policarbonato para blendas com maior conteúdo em policarbonato. Assim sendo, há evidências de que o sistema PC-PS apresenta um grau progressivamente mais baixo de incompatibilidade na medida em que se tem uma maior proporção de policarbonato nas blendas. Tal fato será mais explorado a partir do uso da teoria de Flory-Huggins.

A teoria de Flory-Huggins, desenvolvida para soluções solvente-polímero, pode ser também utilizada para estudar a estabilidade de fase em blendas poliméricas ${ }^{[8-10]}$. A partir dos resultados obtidos da microscopia de infravermelho (Tabela 1), foi possível a determinação do parâmetro de interação polímero-polímero de Flory-Huggins $\left(\chi_{1,2}\right)$. Assumindo-se que a condição de equilíbrio para as blendas foi satisfeita, a equação (1) pode ser derivada a partir da energia-livre de mistura de dois polímeros ${ }^{(8-10]}$ :

$$
\begin{aligned}
\chi_{1,2}=\frac{\left[m_{2} \ln \left(\frac{\varphi_{12}}{\varphi_{11}}\right)+\left(m_{1}-m_{2}\right)\left(\varphi_{21}-\varphi_{22}\right)\right]}{2 m_{1} m_{2}\left(\varphi_{21}^{2}-\varphi_{22}^{2}\right)}+ \\
\frac{\left[m_{1} \ln \left(\frac{\varphi_{22}}{\varphi_{21}}\right)+\left(m_{2}-m_{1}\right)\left(\varphi_{11}-\varphi_{12}\right)\right]}{2 m_{1} m_{2}\left(\varphi_{11}^{2}-\varphi_{12}^{2}\right)}
\end{aligned}
$$

onde $\phi_{11}$ e $\phi_{21}$ são, respectivamente, a fração volumétrica do polímero 1 na fase 1 e do polímero 2 na fase 1 . Da mesma maneira, $\phi_{12}$ e $\phi_{22}$ são, respectivamente, a fração volumétrica do polímero 1 na fase 2 e do polímero 2 na fase 2 . Os valores de $m_{1}$ e $m_{2}$ podem ser considerados iguais ao grau de polimerização dos polímeros 1 e 2. A expressão (1) pode ser usada para calcular o parâmetro de Flory-Huggins $\left(\chi_{1,2}\right)$ para as blendas.

A teoria de Flory-Huggins também possibilita a determinação da condição crítica de estabilidade. O parâmentro crítico de Flory-Huggins $\left(\chi_{1,2}\right)_{\mathrm{c}}$ pode ser calculado como mostrado na equação (2) e define as condições nas quais dissolução e separação de fase vão ocorrer ${ }^{(8-10)}$ :

Tabela 1. Composição das fases de blendas PC-PS avaliadas por micro-FTIR e o parâmetro de interação de Flory polímero-polímero.

\begin{tabular}{cccccc}
\hline $\begin{array}{c}\text { Blendas PS-PC } \\
(\mathbf{e m ~ p e s o})\end{array}$ & $\begin{array}{c}\text { Fase pobre em PS } \\
(\text { Fração vol. de PS)* } \\
\phi_{12}\end{array}$ & $\begin{array}{c}\text { Fase rica em PS } \\
(\text { Fração vol. de PS)* } \\
\phi_{11}\end{array}$ & $\begin{array}{c}\text { \% relativa da fase } \\
\text { rica em PC }\end{array}$ & $\begin{array}{c}\text { \% relativa da fase } \\
\text { rica em PS }\end{array}$ & $\begin{array}{c}\text { Parâmetro Flory- } \\
\text { Huggins }\left(\chi_{1,2}\right)\end{array}$ \\
\hline $25 \%$ PC & 0,11 & 0,87 & 16 & 84 & 0,0190 \\
$50 \%$ PC & 0,17 & 0,86 & 51 & 49 & 0,0181 \\
$75 \%$ PC & 0,19 & 0,80 & 90 & 10 & 0,0167 \\
\hline
\end{tabular}

*Subscritos relativos à fração volumétrica $\phi$ : fase $1=$ fase rica em poliestireno; polímero 1 = poliestireno 


$$
\left(\chi_{1,2}\right)_{c}=\frac{1}{2}\left(\left(m_{1}\right)^{-0.5}+\left(m_{2}\right)^{-0.5}\right)^{2}
$$

A Tabela 1 mostra os parâmetros $\chi_{12}$ para as várias composições da blenda PC-PS investigadas (valores obtidas a partir da aplicação da Equação (1) aos dados da microscopia de infravermelho). Os resultados mostraram que o parâmetro de interação $\chi_{12}$ tende a aumentar na medida em que se tem blendas com maior conteúdo em poliestireno. $\mathrm{O}$ valor crítico de $\chi_{12}$ foi determinado pela Equação (2). Para as blendas PC-PS extrudadas neste trabalho, o valor crítico de $\chi_{12}$ foi igual a 0,0141 . $O$ fato de que os valores de $\chi_{12}$ para as blendas foram mais elevados que o valor de $\left(\chi_{12}\right)_{c}$ é uma indicação de que a blenda PC-PS é imiscível para as composições estudadas. Composições das blendas mais ricas em policarbonato tenderam a deslocar os valores de $\chi_{12}$ para valores mais próximos do parâmetro crítico, sugerindo uma menor incompatibilidade para o sistema com essas composições.

\section{Conclusões}

Este trabalho mostrou que as blendas PC-PS, produzidas por extrusão, apresentam separação de fase clássica, com a presença de uma fase dispersa em uma matriz contínua para composições contendo $25 \%$ dos polímeros e elevada conectividade para blendas com $50 \%$ de cada componente. O uso de técnicas como a microscopia de infravermelho (micro-FTIR) mostrou-se eficiente para detectar os processos de separação de fase, a composição de cada fase nas blendas e determinar o grau de interação entre os componentes da blenda através do parâmetro de interação de FloryHuggins. Os resultados mostraram que blendas com conteúdo mais elevado de policarbonato tendem a apresentar valores de parâmetro de Flory-Huggins mais próximos dos valores críticos, sugerindo maior compatibilidade nessas composições. O conhecimento sobre características relacionadas com a estabilidade de fase de blendas policarbonato-poliestireno (como a composição das fases em equilíbrio e interação entre elas) pode ser usado na definição de compo- sições e condições de processamento capazes de gerar misturas com propriedades óticas controladas.

\section{Agradecimentos}

Os autores agradecem ao CNPq (CTPetro) o auxílio financeiro para execução do trabalho.

\section{Referências Bibliográficas}

1. Chuai, C. Z.; Almdal, K.; Johannsen, Ib. \& LyngaaeJørgensen - J. Polymer, 42, p.8217 (2001).

2. Arrighi, V.; Zajac, W.; Shenoy, S.; Marero, D. M.; Gabrys, B. J.; Garay, M. T.; Gagliardi, S. \& Anderson, K. H. Physica B, 276-278, p.849 (2000).

3. Cabral, J. T.; Gerard, H.; Clarke, N. \& Higgins, J. S. Physica B, 276-278, p.408 (2000).

4. Koh, K. A.; Kim, J. H.; Lee, D. H.; Lee, M. \& Jeong, H. M. - Eur. Polym. J., 34, p. 1229 (1998).

5. Jeon, K. S.; Char, K.; Walsh, D. J. \& Kim, E. - Polymer, 41, p. 2839 (2000).

6. Jang, L. W. \& Lee, D. C. - Polymer, 41, p. 1749 (2000).

7. Kong, Y. \& Hay, J. N. - Polymer, 43, p.1805 (2002).

8. Lipatov, Y. S.; Nesterov, A. E.; Ignatova, T. D.; Nesterov, D. A. - Polymer, 43, p. 875 (2002).

9. Kim, W. N. \& Burns, C. M. - J. Polym. Sci: Polym. Phys., 28, p. 1409 (1990).

10. Kim, W. N. \& Burns, C. M. - J. Appl. Polym. Sci., 34, p. 945 (1987).

Enviado: 03/11/03

Reenviado: 25/03/04

Aprovado: 13/04/04 